\title{
SCSIM: Jointly simulating correlated single-cell and bulk next-generation DNA sequencing data
}

\author{
Collin Giguere ${ }^{1 \dagger}$, Harsh Vardhan Dubey ${ }^{1 \dagger}$, Vishal Kumar Sarsani ${ }^{1 \dagger}$, Hachem Saddiki $^{2}$, Shai He ${ }^{1}$ \\ and Patrick Flaherty ${ }^{*}$ (i)
}

*Correspondence:

flaherty@math.umass.edu

${ }^{\dagger}$ Collin Giguere, Harsh Vardhan

Dubey and Vishal Kumar Sarsani contributed equally to this work.

${ }^{1}$ Department of Mathematics \&

Statistics, University of

Massachusetts Amherst, $710 \mathrm{~N}$.

Pleasant St., 01003 Amherst, USA

Full list of author information is

available at the end of the article

\begin{abstract}
Background: Recently, it has become possible to collect next-generation DNA sequencing data sets that are composed of multiple samples from multiple biological units where each of these samples may be from a single cell or bulk tissue. Yet, there does not yet exist a tool for simulating DNA sequencing data from such a nested sampling arrangement with single-cell and bulk samples so that developers of analysis methods can assess accuracy and precision.
\end{abstract}

Results: We have developed a tool that simulates DNA sequencing data from hierarchically grouped (correlated) samples where each sample is designated bulk or single-cell. Our tool uses a simple configuration file to define the experimental arrangement and can be integrated into software pipelines for testing of variant callers or other genomic tools.

Conclusions: The DNA sequencing data generated by our simulator is representative of real data and integrates seamlessly with standard downstream analysis tools.

Keywords: Single-cell DNA sequencing, simulator, DNA sequencing, Hierarchical Dirichlet

\section{Background}

Simulation software is important for developing and improving statistical methodology for next-generation sequencing data [1]. There are currently 149 such genetic data simulators indexed by the National Cancer Institute [2], and four of these simulators produce DNA sequencing reads with single-nucleotide variants: GemSIM [3], NEAT [4], SInC [5], and CuReSim [6]. Huang et al. [7] proposed one of the first next-generation sequencing (NGS) simulators, but this simulator only generates bulk sequencing data. Gourlé et. al. [8] developed a simulator specifically for metagenomic sequencing experiments. In the past year, two novel simulators for NGS DNA sequencing data have been proposed.

(c) The Author(s). 2020 Open Access This article is licensed under a Creative Commons Attribution 4.0 International License, which permits use, sharing, adaptation, distribution and reproduction in any medium or format, as long as you give appropriate credit to the original author(s) and the source, provide a link to the Creative Commons licence, and indicate if changes were made. The images or other third party material in this article are included in the article's Creative Commons licence, unless indicated otherwise in a credit line to the material. If material is not included in the article's Creative Commons licence and your intended use is not permitted by statutory regulation or exceeds the permitted use, you will need to obtain permission directly from the copyright holder. To view a copy of this licence, visit http://creativecommons.org/licenses/by/4.0/. The Creative Commons Public Domain Dedication waiver (http://creativecommons.org/publicdomain/zero/1.0/) applies to the data made available in this article, unless otherwise stated in a credit line to the data. 
One tool incorporates human population genetic information to simulate structural variation and different types of nucleotide variants [9]. Another tool aims to simulate data from single-cells incorporating allelic dropout, but not false positives or different types of nucleotide variants [10].

The clonal structure of a given sample is difficult to assess from single-cell data alone. Single-cell data suffers from issues like uneven sequencing and partial genome recovery and it is often too expensive to sequence enough single cells to gain a representative sample of the population. On the other hand, bulk samples effectively average over the fine-grain structure present at single-cell level. Developing methods to resolve the clonal populations and their genotypes and determine the relationships between those populations may be advanced by drawing inferences jointly from single-cell and bulk data.

A significant portion of the total sequencing data in existence is generated from experimental studies with model organisms or from repeated measurements of patient samples. These studies have two aspects that are not addressed by existing NGS simulators. First, these datasets contain sequencing data from both bulk tissue or culture as well as single cells. Second, a hierarchical study design induces correlation between samples [11, 12]. For example, an individual cancer patient is sampled from a population, then a tumor is sampled from the individual, and finally, a biopsy is sampled from the tumor. None of the aforementioned simulation tools address these aspects of real datasets.

To address this need, we have developed a software package, single-cell NGS simulator (SCSIM), to allow researchers to simulate bulk and single-cell NGS data from a hierarchical grouped sampling design.

\section{Implementation}

Figure 1 shows a high level workflow diagram of the simulator. The command-line software takes a single haploid reference sequence in FASTA format and a YAML configuration file, and produces FASTQ reads that can be used for downstream alignment and variant calling tasks. The source code can be downloaded from the github repository

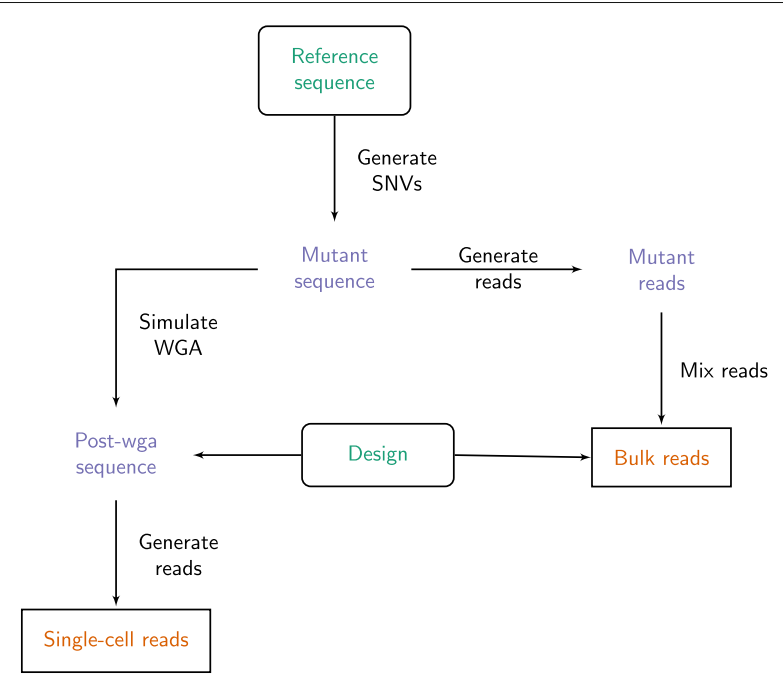

Fig. 1 SCSIM simulation workflow. Inputs (shown in rounded boxes with green text) are the reference sequence and experiment design. Outputs (shown in cornered boxes with orange text) are the bulk and single cell FASTQ read files. Intermediate objects are shown in purple with no boxes 
https://github.com/flahertylab/scsim and the implementation can be run from a docker container defined in the repository.

The nested sampling structure is implemented using a truncated hierarchical Dirichlet mixture model. This model is related to the hierarchical Dirichlet process mixture model in that if the number of components $K \rightarrow \infty$ and the genotypes are drawn i.i.d. from a base measure [13] then the model converges to a hierarchical Dirichlet process mixture model. In sequencing data sets, it is more common to have a fixed number of genotypes, so the Dirichlet mixture model is implemented for this simulator. Errors induced by whole-genome amplification (WGA) of single-cells are simulated using the method described by Zafar et. al. [14]. Then, given the set of diploid reference sequences, NGS reads are simulated using dwgsim [15]. Finally, bulk NGS data is simulated by sampling without replacement from the set of reads from pure samples in proportions defined by the hierarchical Dirichlet model.

\section{Mutated synthetic prototype genome simulation}

Mutated diploid sequences are generated from a single reference (FASTA). Given $K$-the number of mutated synthetic prototype genomes and $n$-the number of possible SNV locations, one-third of SNVs are shared across all mutated sequences, one-third of SNVs are shared across one-half of the mutated sequences, and the remaining one-third of SNVs are shared across a proportion of sequences chosen from a uniform distribution [14]. The set of mutated synthetic prototype genomes is represented by $\boldsymbol{H}$ where $\boldsymbol{h}_{k}$ is a vector of length $n$ containing the mutation location and type information for the $k^{\text {th }}$ mutant genome. The locations of the SNVs are equally spaced across the region of interest in the reference FASTA. Given the status of each SNV location in each mutated sequence, the type of diploid mutation (heterozygous or homozygous) and base substitutions are generated according to a transition probability matrix derived from Pattnaik et. al. (SiNC) [5]. The transition probabilities, and SNV locations can be set by the user, and have default values derived from literature.

\section{Hierarchical sampling model}

We define a biological unit as the top-level sampling unit. For example, in cancer sequencing a biological unit may be an individual patient, or, in experimental model organism sequencing, a biological unit may be a biological replicate. A sample is the bottom-level sampling unit. In cancer sequencing, a sample may be a single-cell or bulk biopsy, or, in experimental model organism sequencing, a sample may be a technical replicate.

A hierarchical Dirichlet model is used to simulate correlation between samples. First, the population distribution over mutated synthetic prototype genomes, $G^{\prime \prime}$, is sampled from a Dirichlet distribution with parameter $\boldsymbol{\alpha}$. Then, the distribution over mutated sequences for biological unit $i, \boldsymbol{G}^{\prime}$, is sampled from a Dirichlet distribution with parameter $\beta_{i} \boldsymbol{G}^{\prime \prime}$ where $\beta_{i}$ controls the concentration of the distribution of the biological unit around the population distribution. Finally, the distribution over mutated sequences for sample $j$ in biological unit $i, \boldsymbol{G}_{i j}^{\prime}$, is distributed as a Dirichlet with parameter $\gamma_{i j} \boldsymbol{G}^{\prime}{ }_{i}$ where $\gamma_{i j}$ controls the concentration of the sample around the biological unit distribution. The distributions, $\boldsymbol{G}^{\prime \prime}, \boldsymbol{G}^{\prime}{ }_{i}$, and $\boldsymbol{G}^{\prime}{ }_{i j}$ are all $K$-dimensional vectors because there are $K$ mutant synthetic genomes in $\boldsymbol{H}$. 
The hierarchical Dirichlet generative model is summarized as

$$
\begin{array}{rlr}
\boldsymbol{G}^{\prime \prime} & \sim \operatorname{Dirichlet}(\boldsymbol{\alpha}), \\
\boldsymbol{G}^{\prime}{ }_{i} & \sim \operatorname{Dirichlet}\left(\beta_{i} \boldsymbol{G}^{\prime \prime}\right), & \\
\boldsymbol{G}_{i j} & \sim \operatorname{Dirichlet}\left(\gamma_{i j} \boldsymbol{G}^{\prime}{ }_{i}\right), & \text { for } i=1, \ldots, N \\
\boldsymbol{H}_{k} & \sim \operatorname{SiNC}(\theta), & \text { for } i=1, \ldots, N, \quad j=1, \ldots, N_{i} \\
& \text { for } k=1, \ldots, K
\end{array}
$$

where SiNC is the model for generating variant locations and types which depends on parameter $\theta$ [5]. Figure 2 shows a graphical model representation of Model 1 where $\boldsymbol{X}_{i j}$ are the reads generated according to the bulk and single-cell sampling models.

To ground the sampling model in a real data example, we consider a cancer sequencing dataset. First, a distribution over cancer genotypes is sampled from $G^{\prime \prime}$. Then, for each individual patient (biological unit), $i$, a distribution over cancer genotypes, $\boldsymbol{G}^{\prime}{ }_{i}$, is sampled. This distribution represents the fraction of the total tumor burden in the individual from each clonal genotype. Finally, for each biopsy (sample), $j$, a distribution over cancer genotypes, $\boldsymbol{G}_{i j}$, is sampled. This distribution represents the fraction of the bulk or single-cell biopsy from each clonal genotype. Clearly, the distribution over genotypes for single-cell biopsy should be concentrated at a single genotype; whereas the distribution for a bulk biopsy may be concentrated at a single genotype or more diffuse if the biopsy

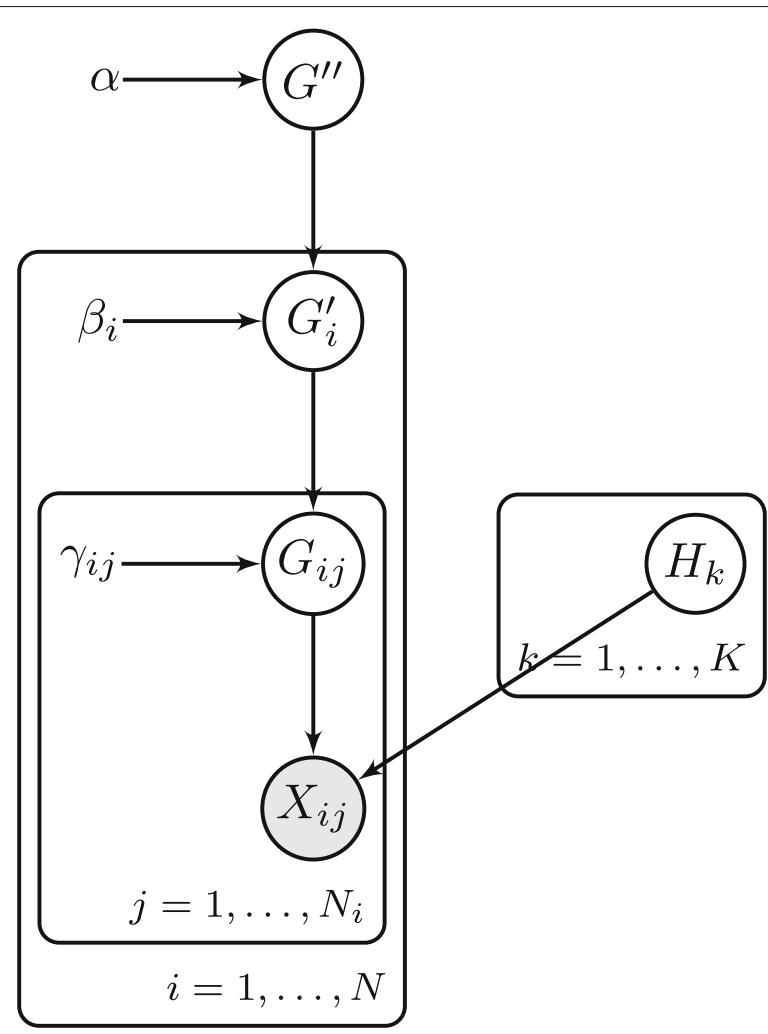

Fig. 2 Graphical model representation of the SCSIM hierarchical model. To generate data from the model, first, sample a distribution over $K$ mutant genomes, $\boldsymbol{G}^{\prime \prime}$. Sample a distribution for each biological unit (individual), $\boldsymbol{G}_{i}^{\prime}$ for $i=1, \ldots, N$. Sample a distribution for each sample (biopsy), $\boldsymbol{G}_{i j}^{\prime}$ for $j=1, \ldots, N_{i}$. Sample variant locations and types that define the mutant synthetic genomes $\boldsymbol{H}=\left[\boldsymbol{h}_{1}, \ldots, \boldsymbol{h}_{K}\right]$. Finally, sample the bulk and single-cell reads for each sample $\boldsymbol{X}_{i j}$ 
is heterogeneous. The hierarchical Dirichlet model allows our simulator to generate data from exactly this experimental sampling structure.

\section{Sampling models}

The observed reads for sample $(i, j), \boldsymbol{X}_{i j}$, are generated differently for bulk and single-cell samples. The total number of samples for biological unit $i$ is the summation of the singlecell samples and the bulk samples, $N_{i}=N_{i}^{\mathrm{sc}}+N_{i}^{\mathrm{bu}}$. We describe the single-cell and bulk read sampling models next.

Single-cell data sampling model For each of the $N_{i}^{\mathrm{SC}}$ single-cell samples, a wholegenome amplification (WGA) model is applied to the corresponding mutated sequence. Allelic dropout (ADO) and false positive (FP) mutations from the WGA process were generated as done previously [14]. The ADO rate was set to $20 \%$ and the FP rate was set to $3.2 \times 10^{-5}$ [16]. The ADO and FP rates are calculated in reference to the entire length of the reference sequence. Finally, sequencing reads and corresponding FASTQ files were generated with dwgsim for each of the $N_{s c}$ single-cell samples.

Bulk data sampling model First dwgsim was used to generate FASTQ reads for each of the $K$ mutated synthetic prototype genomes. Then for each of the $N_{i}^{\text {bu }}$ bulk samples, the FASTQ reads from each of the mutated synthetic prototype genomes were mixed according to the sample distribution $G_{i j}$.

\section{Results}

Here we test whether the output generated by SCSIM are consistent with the output generated by real experiments. We say that the output is consistent when the FASTQ read files generated by SCSIM produce variant calls that are comparable to variant calls produced from FASTQ files from real experiments.

Simulation protocol In order to assess the consistency of reads simulated by SCSIM, we used the results from two variant callers: BCFtools [17] and Monovar [14]. We measure the accuracy of the variant callers in terms of (1) true positive rate or recall and (2) positive predictive value or precision.

We extracted a 1 million base pair region from hg38 starting at chr20:100000. We generated three diploid synthetic prototype genomes each with 100 potential SNVs spaced every 8080 base pairs. The zygosity of the SNVs was sampled according to the method described in the "Implementation" section. The prior parameter for the Dirichlet distribution across mutated synthetic prototype genomes, $\alpha$ was set to $(0.1,0.3,0.6)$. Figure 3 shows the distribution of true SNVs across genomic position for each mutated synthetic prototype genome. The concentration parameter for the Dirichlet distribution at the biological unit level $\beta_{i}$, was set to 0.1 for all $i$. The concentration parameter for the Dirichlet distribution for samples within biological units, $\gamma_{i j}$ was set to 0.1 for all $(i, j)$. Two samples were generated for each unit with unit 1 and 4 having one bulk and one single-cell sample, unit 2 having two single-cell samples, and unit 3 having 2 bulk samples. The mean coverage level was set to $24 \times$. For bulk samples, we drew 1,000,000 reads according to the distribution over prototypes that was realized by the hierarchical Dirichlet model. A 


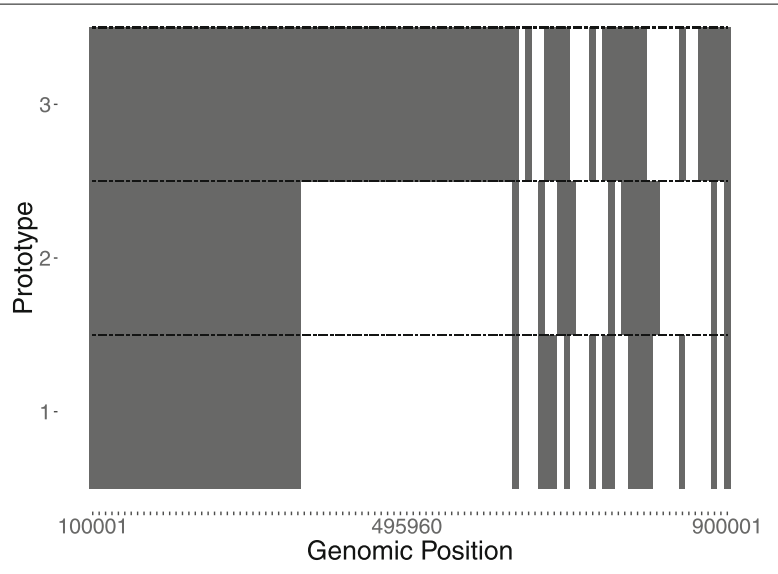

Fig. 3 Simulated true SNV locations across mutated synthetic prototype genomes

total of 90 SNVs introduced across 4 single-cell and 4 bulk samples from the described simulation protocol are the true SNVs and served as the gold standard set.

Visualization of simulated reads The simulated FASTQ reads of single-cell and bulk samples across 4 biological units were mapped to the human genome assembly (hg38) using the Burrows-Wheeler alignment tool [18]. Figure 4 shows IGV visualizations of three genomic locations with varying proportions of samples with true SNVs.

We compared the quality metrics of SNVs detected from simulated reads to the four different human acute lymphoblastic leukemia patients that were previously published [19]. That study collected both single-cells and bulk samples of six patients to better understand genomic heterogeneity. A 1-Mbp region of chromosome 20 of the human genome (hg38) was chosen as the reference genome. Both simulated reads and the patient data were aligned and variants were called on $1-\mathrm{Mbp}$ region. The $\mathrm{Ti} / \mathrm{Tv}$ (transition to transversion) ratio of both simulated and patient data fall within the range of 1.2-1.6. We also compared substitutions percentages across the simulated reads and the patient data. While most substitutions of the patient and simulated data fall within the comparable range, less than expected $\mathrm{T}>\mathrm{A}$ substitutions in our patient data and $\mathrm{C}>\mathrm{A}$ in the simulated data. This can be attributed to the nature of single-cell data which suffers from issues like uneven sequencing, coverage, and partial genome recovery.

Mapped reads were used to call variants by Monovar [14] and BCFtools [17], two popular SNV callers used for the single-cell data. Monovar and BCFtools were run with default parameter values on the BAM files of all single-cell and bulk data.

BCFtools and Monovar called 154 and 156 SNVs respectively across 4 single-cell and 4 bulk simulated samples. Our analysis showed that out of 90 true SNVs, 88 were called by both BCFtools and Monovar resulting in a recall of $97.77 \%$ for both methods. BCFtools had a precision of $57.1 \%$ and Monovar had a precision of $56.4 \%$. Previous research has shown that variant calls from BCFtools and Monovar tend to have high sensitivity and low specificity (see [14] Supplementary Figure 3 and [20]). Low specificity may be acceptable if the variant calls are heavily filtered to reduce the final false positive rate. Figure 5 shows a Venn diagram of the concordance between true SNVs and called SNVs from BCFtools and Monovar. 


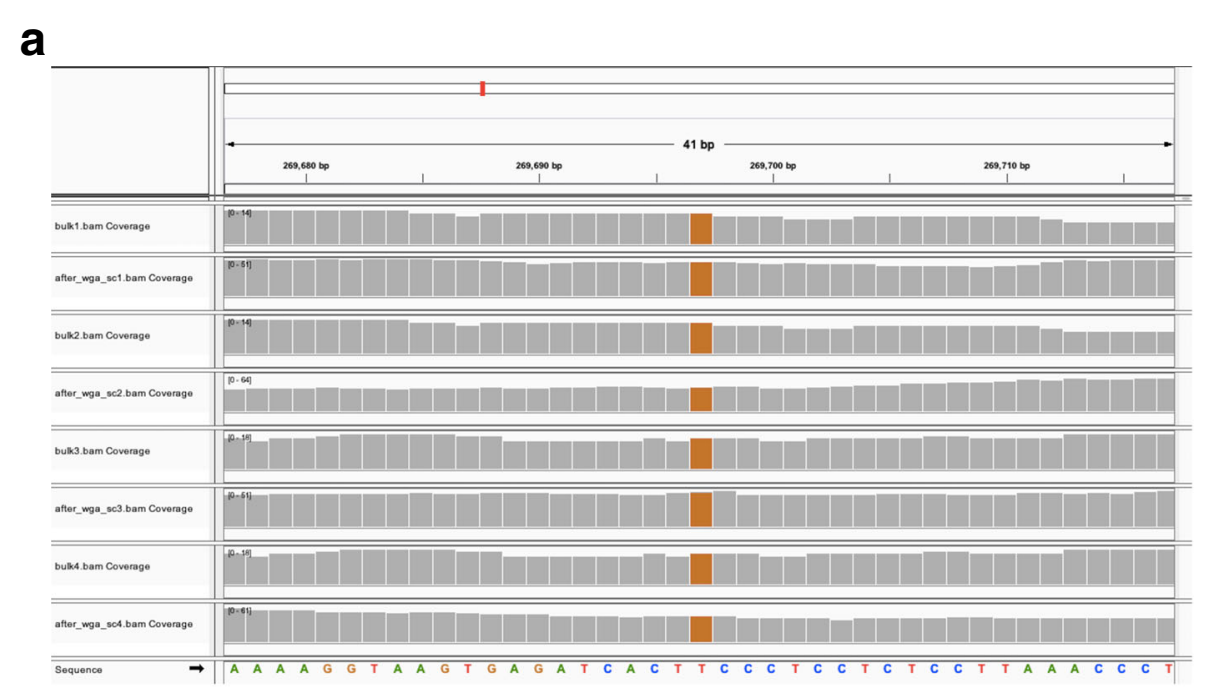

b

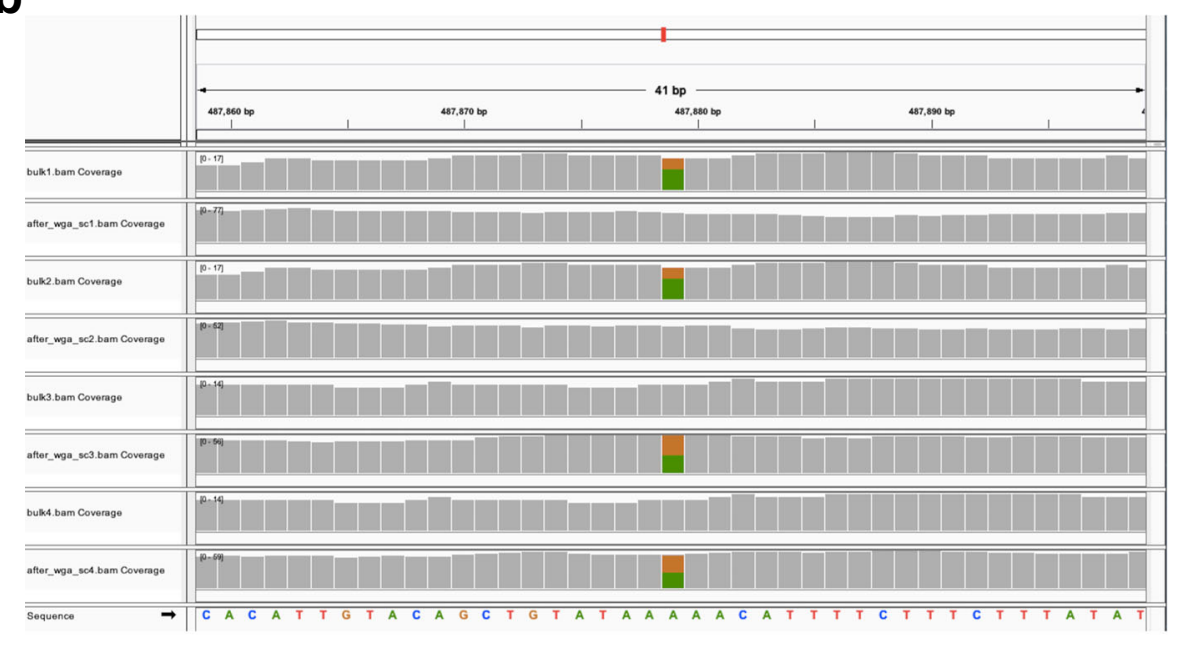

C

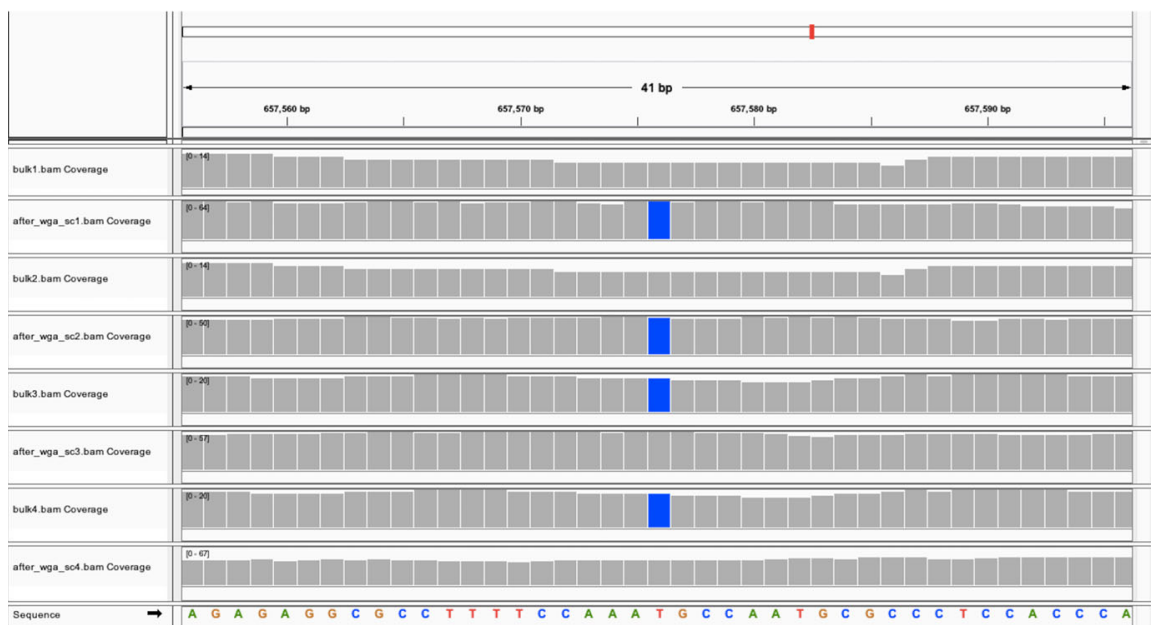

Fig. 4 IGV visualization of reads generated by SCSIM for 4 single-cell and 4 bulk samples at $\mathbf{a}$ a genomic location where each sample has a true SNV, $\mathbf{b}$ at a genomic location where half of the samples have a true SNV, and $\mathbf{c}$ at a genomic location where a random fraction of the samples have a true SNV 


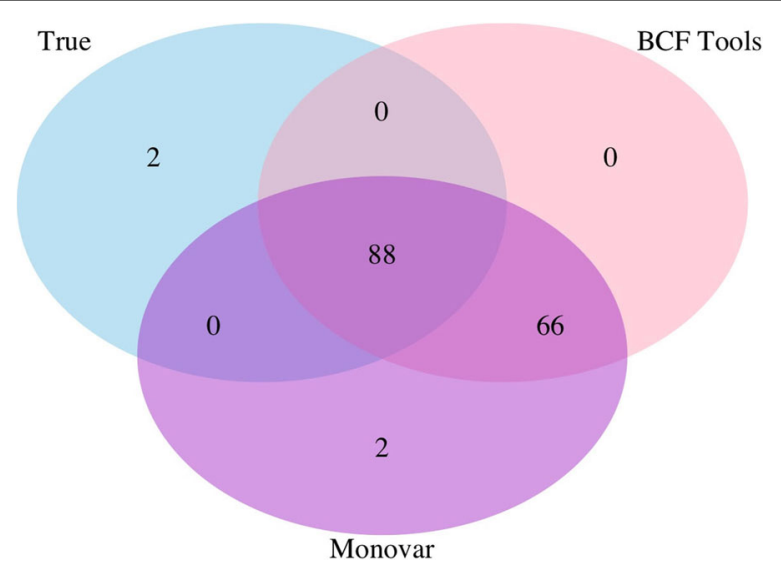

Fig. 5 Venn diagram showing concordance of called and true variants

It is common to post-filter the variant calls to increase specificity at the expense of decreased sensitivity. We applied the following standard post-filtration steps to the variant calls from Monovar and BCFtools: (1) minimum mapping quality of reads (>1), (2) minimum base quality ( $>30)$, (3) minimum number of variant-supporting reads $(>5)$, (4) remove strand biasedness. As expected, after filtration, recall decreased, and precision increased. The F1-score, a weighted measure of precision and recall, for both Monovar and BCFtools is 0.779 . In comparison, the F1-score before filtration is 0.715 for Monovar and 0.725 for BCFtools. These F1-scores are in-line with previously reported analysis on real and simulated data which is consistent with the total number of reads across all samples indicating that our simulation tool provides FASTQ reads consistent with real data $[14,21]$.

\section{Conclusions}

Given the increase in the amount of single-cell next-generation DNA sequencing data there is a need for reproducible bioinformatics methods for performing statistical inference on that data. To our knowledge there are no methods for jointly simulating bulk and single-cell sequencing data, yet these simulation tools are needed to test and validate inference methods. SCSIM jointly simulates bulk and single-cell next-generation sequencing data and generates correlated samples using a hierarchical truncated Dirichlet distribution for sampling the distribution over mutant sequences for bulk samples. Our implementation, using a docker container, allows it to be inserted in a bioinformatics pipeline without modifying existing dependencies.

\section{Availability and requirements}

Project name: single-cell DNA sequencing data simulator

Project home page: https://github.com/flahertylab/scsim

Operating systems(s): Any

Programming language: Python

Other requirements: docker

License: MIT

Any restrictions to use by non-academics: none 


\section{Abbreviations}

ADO: allelic dropout; FP: false positive; SCSIM: single-cell NGS simulator; NGS: next-generation sequencing; WGA: whole-genome amplification

\section{Acknowledgements}

Not applicable.

\section{Authors' contributions}

CG contributed to the development of the algorithm and implementation and drafting of the manuscript. HVD contributed to the implementation of the algorithm, analysis and interpretation of the data, and drafting of the manuscript. VKS contributed to the analysis and interpretation of the data and drafting of the manuscript. HS contributed to the development of the algorithm. SH contributed to the implementation of the algorithm and drafting of the manuscript. PF contributed to the study conception and design, development of the algorithm, analysis and interpretation of the data and drafting of the manuscript. All author(s) have read and approved the manuscript.

\section{Funding}

The analysis, interpretation of data, and writing of the manuscript was funded in part by NIH award 1R01GM135931-01. The funding body did not play a role in the design of the study and collection, analysis, and interpretation of data and in writing the manuscript.

\section{Availability of data and materials}

The data and materials used in this study are openly available at the locations described in the relevant sections.

\section{Ethics approval and consent to participate}

Not applicable

\section{Consent for publication}

Not applicable

\section{Competing interests}

The authors declare that they have no competing interests.

\section{Author details}

${ }^{1}$ Department of Mathematics \& Statistics, University of Massachusetts Amherst, 710 N. Pleasant St., 01003 Amherst, USA.

${ }^{2}$ School of Public Health, University of Massachusetts Amherst, 01003 Amherst USA.

\section{Received: 3 February 2020 Accepted: 18 May 2020}

Published online: 26 May 2020

\section{References}

1. Escalona M, Rocha S, Posada D. A comparison of tools for the simulation of genomic next-generation sequencing data. Nat Rev Genet. 2016;17(8):459-69. https://doi.org/10.1038/nrg.2016.57.

2. NCl Division of Cancer Control \& Population Sciences. Genetic Simulation Resources. 2018. https://popmodels. cancercontrol.cancer.gov/gsr/. Accessed 27 Nov 2018.

3. McElroy KE, Luciani F, Thomas T. GEMSIM: General, error-model based simulator of next-generation sequencing data. BMC Genomics. 2012;13(1):74. https://doi.org/10.1186/1471-2164-13-74.

4. Stephens ZD, Hudson ME, Mainzer LS, Taschuk M, Weber MR, lyer RK. Simulating next-generation sequencing datasets from empirical mutation and sequencing models. PLOS ONE. 2016;11(11):0167047. https://doi.org/10. 1371/journal.pone.0167047.

5. Pattnaik S, Gupta S, Rao AA, Panda B. SInC: an accurate and fast error-model based simulator for SNPs, indels and CNVs coupled with a read generator for short-read sequence data. BMC Bioinformatics. 2014;15(1):40. https://doi. org/10.1186/1471-2105-15-40.

6. Caboche S, Audebert $C$, Lemoine $Y$, Hot D. Comparison of mapping algorithms used in high-throughput sequencing: Application to ion torrent data. BMC Genomics. 2014;15(1):264. https://doi.org/10.1186/1471-2164-15264.

7. Huang W, Li L, Myers JR, Marth GT. Art: a next-generation sequencing read simulator. Bioinformatics. 2012;28(4): 593-4.

8. Gourlé H, Karlsson-Lindsjö O, Hayer J, Bongcam-Rudloff E. Simulating Illumina metagenomic data with InSilicoSeq. Bioinformatics. 2018;35(3):521-2. https://doi.org/10.1093/bioinformatics/bty630 https://academic.oup.com/ bioinformatics/article-pdf/35/3/521/27699758/bty630.pdf.

9. Wang S, Wang J, Xiao X, Zhang X, Wang X, Zhu X, Lai X. GSDcreator: An Efficient and Comprehensive Simulator for Genarating NGS Data with Population Genetic Information. In: 2019 IEEE International Conference on Bioinformatics and Biomedicine (BIBM). San Diego: IEEE; 2019. p. 1868-75. https://doi.org/10.1109/BIBM47256.2019.8983192.

10. Yu Z, Du F, Sun X, Li A. SCSsim: an integrated tool for simulating single-cell genome sequencing data. Bioinformatics. 2019;36(4):1281-2. https://doi.org/10.1093/bioinformatics/btz713 https://academic.oup.com/ bioinformatics/article-pdf/36/4/1281/32527663/btz713.pdf.

11. Casasent AK, Schalck A, Gao R, Sei E, Long A, Pangburn W, Casasent T, Meric-Bernstam F, Edgerton ME, Navin NE. Multiclonal invasion in breast tumors identified by topographic single cell sequencing. Cell. 2018;172(1-2): 205-21712. https://doi.org/10.1016/j.cell.2017.12.007.

12. Zhou J, Deng Y, Shen L, Wen C, Yan Q, Ning D, Qin Y, Xue K, Wu L, He Z, Voordeckers JW, Nostrand JDV, Buzzard V, Michaletz ST, Enquist BJ, Weiser MD, Kaspari M, Waide R, Yang Y, Brown JH. Temperature mediates 
continental-scale diversity of microbes in forest soils. Nat Commun. 2016;7(1):12083. https://doi.org/10.1038/ ncomms12083.

13. Teh YW, Jordan MI, Beal MJ, Blei DM. Hierarchical dirichlet processes. J Am Stat Assoc. 2006;101(476):1566-81. https://doi.org/10.1198/016214506000000302.

14. Zafar H, Wang Y, Nakhleh L, Navin N, Chen K. Monovar: single-nucleotide variant detection in single cells. Nat Methods. 2016;13(6):505-507. https://doi.org/10.1038/nmeth.3835.

15. Homer N. Whole Genome Simulator for Next-Generation Sequencing. 2018. http://github.com/nh13/dwgsim. Accessed 27 Nov 2018

16. Leung ML, Wang Y, Waters J, Navin NE. SNES: single nucleus exome sequencing. Genome Biol. 2015;16(1):55. https://doi.org/10.1186/s13059-015-0616-2.

17. Li H. A statistical framework for SNP calling, mutation discovery, association mapping and population genetical parameter estimation from sequencing data. Bioinformatics. 2011;27(21):2987-93. https://doi.org/10.1093/ bioinformatics/btr509.

18. Li H, Durbin R. Fast and accurate short read alignment with Burrows-Wheeler transform. Bioinformatics. 2009;25(14): 1754-60. https://doi.org/10.1093/bioinformatics/btp324 https://academic.oup.com/bioinformatics/article-pdf/25/ 14/1754/605544/btp324.pdf.

19. Gawad C, Koh W, Quake SR. Dissecting the clonal origins of childhood acute lymphoblastic leukemia by single-cell genomics. Proc Natl Acad Sci. 2014;111(50):17947-52. https://doi.org/10.1073/pnas.1420822111 https://www.pnas. org/content/111/50/17947.full.pdf.

20. Bowles NE, Jou CJ, Arrington CB, Kennedy BJ, Earl A, Matsunami N, Meyers LL, Etheridge SP, Saarel EV, Bleyl SB, Yost HJ, Yandell M, Leppert MF, Tristani-Firouzi M, Gruber PJ. the Baylor Hopkins Centers for Mendelian Genomics: Exome analysis of a family with wolff-parkinson-white syndrome identifies a novel disease locus. Am J Med Genet A. 2015;167(12):2975-84. https://doi.org/10.1002/ajmg.a.37297.

21. Kumaran M, Subramanian U, Devarajan B. Performance assessment of variant calling pipelines using human whole exome sequencing and simulated data. BMC Bioinformatics. 2019;20(1):342. https://doi.org/10.1186/s12859-0192928-9.

\section{Publisher's Note}

Springer Nature remains neutral with regard to jurisdictional claims in published maps and institutional affiliations.

\section{Ready to submit your research? Choose BMC and benefit from:}

- fast, convenient online submission

- thorough peer review by experienced researchers in your field

- rapid publication on acceptance

- support for research data, including large and complex data types

- gold Open Access which fosters wider collaboration and increased citations

- maximum visibility for your research: over $100 \mathrm{M}$ website views per year

At $B M C$, research is always in progress.

Learn more biomedcentral.com/submissions 